BASIC SCIENCE ARTICLE

\title{
New insights into neonatal coagulation: normal clot formation despite lower intra-clot thrombin levels
}

\author{
Harald Haidl ${ }^{1}$, Evelyn Zöhrer ${ }^{1}$, Sina Pohl ${ }^{1}$, Bettina Leschnik ${ }^{1}$, Eva-Christine Weiss ${ }^{2}$, Siegfried Gallistl ${ }^{1}$, Wolfgang Muntean ${ }^{1}$ and \\ Axel Schlagenhauf ${ }^{1}$
}

\begin{abstract}
BACKGROUND: Healthy neonates exhibit no bleeding tendencies, but exhibit longer partial thromboplastin times than adults. Lower clotting factor levels may be balanced by lower inhibitor levels, which is not reflected in routine coagulation assays, but could result in normal clot formation in vivo. The novel thrombodynamics assay simulates a damaged vessel with tissue factor immobilized to a surface. We hypothesized that intra-clot thrombin levels and spatial fibrin clot formation with this assay are comparable in neonates and adults.

METHODS: Coagulation was tested in plasma from venous neonatal blood $(N=12)$, cord blood $(N=30)$, and adult blood $(N=20)$ using thrombodynamics and calibrated automated thrombography.

RESULTS: Neonates exhibited a higher initial rate of clot formation than adults (adult: $60.7 \pm 3.9 \mu \mathrm{m} / \mathrm{min}$; neonatal: $66.8 \pm 3.9 \mu \mathrm{m} /$ $\mathrm{min}$; cord: $68.1 \pm 3.3 \mu \mathrm{m} / \mathrm{min} ; P<0.001$ ) and a comparable stationary rate of clot formation (adult: $35.8 \pm 8.5 \mu \mathrm{m} / \mathrm{min}$; neonatal: $37.0 \pm 4.6 \mu \mathrm{m} / \mathrm{min}$; cord: $36.0 \pm 5.2 \mu \mathrm{m} / \mathrm{min} ; P=0.834$ ). Intra-clot thrombin levels were lower in neonates (adult: $41.9 \pm 11.2 \mathrm{AU} /$; neonatal: $22.6 \pm 10.2 \mathrm{AU} / \mathrm{l}$; cord: $23.6 \pm 9.7 \mathrm{AU} / \mathrm{l} ; P<0.001$ ), but the longitudinal rate of thrombin propagation was comparable (adult: $27.2 \pm 4.2 \mu \mathrm{m} / \mathrm{min}$ neonatal; $27.9 \pm 2.9 \mu \mathrm{m} / \mathrm{min}$; cord: $27.6 \pm 3.4 \mu \mathrm{m} / \mathrm{min} ; P=0.862$ ).

CONCLUSIONS: Despite lower intra-clot thrombin levels, neonates exhibit normal spatial fibrin clot growth, which concurs with
\end{abstract} clinically well-functioning hemostasis in healthy neonates.

Pediatric Research (2019) 86:719-724; https://doi.org/10.1038/s41390-019-0531-4

\section{INTRODUCTION}

Healthy neonates show no clinical signs of coagulation abnormalities. Neonates exhibit neither easy bruising nor bleeding tendencies. ${ }^{1-4}$ However, the neonatal hemostatic system exhibits lower levels of several vitamin K-dependent and -independent clotting factors (factor II (FII), IX, X, XI, and XII), which is reflected in a prolonged activated partial thromboplastin time (aPTT). ${ }^{5}$ The discrepancy between prolonged aPTT and a normal clinical picture can be explained by lower levels of coagulation inhibitors (antithrombin, protein $\mathrm{C}$, and protein S) and elevated levels of a2-macroglobulin, which additionally inhibits the protein C/ protein $\mathrm{S}$ system. ${ }^{6,7}$ Lower levels of tissue factor pathway inhibitor (TFPI) result in a higher fraction of thrombin generated via tissue factor/factor Vlla than in adults, which explains why neonates with hemophilia exhibit lower bleeding tendencies than later in life. ${ }^{8}$ Taken together, lower inhibitor activity compensates for lower levels of clotting factors in vivo. Hence, the hemostatic system of neonates is balanced at a lower level than that of adults and exhibits a higher fragility.

However, this peculiarity is not accurately reflected in most in vitro tests. There is a need for functional assays that accurately depict the clinical picture of neonatal hemostasis despite its peculiarities. Furthermore, detection of subtle shifts in the neonatal hemostatic balance are required to allow differentiation of healthy and pathological conditions. The aPTT is not suitable to accurately depict hemostasis of healthy neonates because this assay employs high concentrations of contact phase-activating agents (e.g., kaolin), resulting in low influence of varying inhibitor levels on the clotting time. ${ }^{10}$ Assays taking primary hemostasis into account (e.g., PFA100 and thromboelastography) seem to accurately depict neonatal coagulation, but lack specificity for various pathological conditions in secondary hemostasis. ${ }^{11,12}$

A more accurate measure for the hemostatic balance is the amount of thrombin generated over time as $90 \%$ are generated after the onset of clot formation. ${ }^{13,14}$ Thrombin generation can be traced in vitro using calibrated automated thrombography (CAT). To our knowledge, studies on neonatal hemostasis with this assay were exclusively performed with cord blood as a surrogate for venous neonatal blood, because ample amounts of plasma are required. The endogenous thrombin potential (ETP) calculated from the measurement corresponds to the overall amount of thrombin generated in the plasma. Accordance of the ETP with the clinical picture of neonates depends on the employed tissue factor concentration. At commonly employed tissue factor concentrations $(5 \mathrm{pM})$, the ETP in samples from cord blood is about $50 \%$ of that in adults. ${ }^{15}$ The assay is more sensitive to feedback loops and inhibitors with lower tissue factor concentrations $(1 \mathrm{pM}) .^{16}$ In this setting, the ETP from cord blood equals that of adults. ${ }^{15}$

However, there is no correct tissue factor concentration to optimally depict in vivo conditions, since tissue factor is usually not evenly distributed in the sample, but located on a cell surface.

\footnotetext{
${ }^{1}$ Department of General Pediatrics and Adolescent Medicine, Medical University of Graz, Graz, Austria and ${ }^{2}$ Department of Obstetrics and Gynaecology, Medical University of Graz, Graz, Austria

Correspondence: Axel Schlagenhauf (axel.schlagenhauf@medunigraz.at)
}

Received: 3 January 2019 Revised: 1 August 2019 Accepted: 2 August 2019

Published online: 12 August 2019 
According to the cell-based model of hemostasis tissue factordependent and -independent processes are locally separated on different cell surfaces in vivo. ${ }^{17}$ In the initiation phase of this model, a small amount of thrombin is formed on a tissue factor bearing cell via the extrinsic pathway. In the propagation phase, this small amount of thrombin creates a thrombin burst on platelets without tissue factor via the factor XI feedback loop and intrinsic components of the coagulation system. The concept of those two phases being locally separated is not reflected in any assay wherein tissue factor is added and homogeneously distributed in plasma (prothrombin time (PT), CAT, thromboelastrography, etc.).

The novel thrombodynamics assay is designed to simulate a damaged vessel wall. Coagulation is triggered with tissue factor immobilized on a surface, which allows evaluation of tissue factordependent and -independent clot formation in local separation. ${ }^{18,19}$ The rate of spatial clot formation is calculated by optically tracing the clot front. Furthermore, the generation and distribution of thrombin within the clot is measured with a fluorescent substrate. This in vitro assay should accurately depict neonatal coagulation as it closely resembles in vivo conditions while retaining sensitivity to varying inhibitor levels.

Koltsova et al. ${ }^{20}$ investigated spatial fibrin clot formation and platelet function in preterm versus term neonates at days 1 and 4 after delivery. ${ }^{20}$ In contrast to routine parameters, they found hypercoagulation in plasmatic coagulation of term as well as preterm neonates paired with reduced platelet function, which both is in line with our previous studies. ${ }^{21,22}$ However, Koltsova et al. $^{20}$ did not investigate thrombin generation and intra-clot thrombin levels in their cohorts.

Considering the clinical picture of normal coagulation in neonates and our previous investigations, we hypothesized that, despite lower in vitro thrombin generation with the thrombin generation assay, intra-clot thrombin levels are similar in neonates and adults. We wanted to test this hypothesis in a comparative study with venous neonatal blood, cord blood, and adult blood using CAT and the thrombodynamics assay.

\section{METHODS}

Participants and preanalytics

This study was carried out in accordance with the recommendations of the Ethics Committee of the Medical University of Graz, Austria (EK-No. 27-100 ex 14/15). All adult participants gave written informed consent in accordance with the Declaration of Helsinki. All mothers gave written informed consent for the procurement of cord blood or venous blood. The protocol was approved by the Ethics Committee of the Medical University of Graz.

Venous neonatal blood was obtained from full-term infants with uncomplicated delivery after 39-42 weeks of gestation during follow-up of prolonged neonatal jaundice. Hemostasis in these infants was clinically inconspicuous; they showed no signs of bruising or thrombosis. All neonates received vitamin $\mathrm{K}$ postpartum before the samples were taken. Cord blood was obtained from healthy full-term neonates, following uncomplicated delivery after 39-42 weeks of gestation. Demographic data of the neonatal cohorts are depicted in Table 1. Venous blood was obtained from self-reported healthy adults with no regular medication and no antithrombotic or antiplatelet therapy for the last 4 weeks. Demographic data of the adult cohort are depicted in Table S1.

Blood was drawn with a 21-gauge needle from the antecubital vein in adults, without applying venostasis, from the umbilical cord after separation of the placenta, and from a scalp vein in infants. All samples were collected in pre-citrated S-Monovette premarked vacuum tubes from Sarstedt containing $0.106 \mathrm{~mol} / \mathrm{l}$ trisodium citrate solution (adult and cord blood: $3.0 \mathrm{ml}$ tubes; venous neonatal blood: $1.4 \mathrm{ml}$ tubes). Platelet-poor plasma was
Table 1. Demographic information of the neonatal study cohorts

\begin{tabular}{lcl}
\hline & Venous neonatal blood & Cord blood \\
\hline$N$ (female/male) & $12(6 / 6)$ & $30(16 / 14)$ \\
Gestational age [weeks] & $40(39-42)$ & $40(39-42)$ \\
Postnatal age [days] & $21(8-30)$ & - \\
Birthweight $[\mathrm{g}]$ & $3052(2901-3725)$ & $3123(2981-3839)$ \\
Hematocrit [\%] & $38.2(30.8-43.6)$ & $36.3(27.6-43.1)$ \\
Platelet count $\left[\times 10^{3} / \mu \mathrm{l}\right]$ & $408(208-612)$ & $223(166-271)^{* *}$ \\
Total bilirubin $[\mathrm{mg} / \mathrm{dl}]$ & $10.3(4.9-14.8)$ & - \\
\hline
\end{tabular}

Data are depicted in median (range). Asterisks indicate significant differences between the cohorts tested with Mann-Whitney U-test ${ }^{* * P} P$ 0.001)

obtained immediately by centrifugation $(1600 \times g, 15 \mathrm{~min})$, and then recentrifuged $(10,000 \times g, 5 \mathrm{~min})$ before samples were snap frozen at $-80^{\circ} \mathrm{C}$. Samples were thawed at $37^{\circ} \mathrm{C}$ for $5 \mathrm{~min}$ before analyses. All samples were subjected to measurements with the thrombodynamics assay and routine coagulation assays. Cord blood and adult samples were also subjected to thrombin generation measurements with CAT. Venous neonatal samples were excluded from CAT measurements due to insufficient sample material.

\section{Thrombodynamics assay}

Spatial fibrin clot formation in plasma samples was measured on a Thrombodynamics Analyzer System T2-T analyzer using the Thrombodynamics-4D reagent kit (Hemacore, Moscow, Russia). Samples were thawed, corn trypsin inhibitor $(0.2 \mathrm{mg} / \mathrm{ml})$ and the fluorescent substrate Z-Gly-Gly-Arg-AMC $(400 \mu \mathrm{M})$ were added to $120 \mu \mathrm{l}$ plasma, and the mixture was incubated at $37^{\circ} \mathrm{C}$ for $15 \mathrm{~min}$ to prevent contact phase activation. Calcium acetate $(20 \mathrm{mM})$ and phospholipids ( $4 \mu \mathrm{M}$, phosphatidyl serine/choline 20:80 molar ratio) were added to the samples immediately before the measurement. Sample mixtures were transferred into transparent cuvettes, placed in the analyzer, and kept at $37^{\circ} \mathrm{C}$. Clot formation was started by positioning a tissue factor bearing insert $\left(90 \mathrm{pmol} / \mathrm{m}^{2}\right)$ into the cuvette. The measurement chamber was kept at $37^{\circ} \mathrm{C}$ and $0.02 \mathrm{~atm}$. Clot formation originating from the insert was monitored optically by continuously tracing the clot front for $60 \mathrm{~min}$. The distance between insert and clot front was translated into clot thickness via internal standardization and plotted against time. Several parameters were determined from this plot: the lag time corresponds to the time between placement of the insert and onset of fibrin clot formation. The initial rate of clot formation $\left(V_{\mathrm{i}}\right)$ corresponds to the slope of the clot front trace in the interval 2-6 min after onset of clot formation and the stationary rate $\left(V_{s}\right)$ in the interval $15-25 \mathrm{~min}$. The clot density was determined by optically detecting the intensity of light scattering and depicted in arbitrary units (AU).

Generation of cleaved fluorescent thrombin substrate was monitored as well. Thrombin distribution within the clot was traced based on changes in fluorescence intensities. It has been shown that thrombin propagates similar to a wave with a stationary amplitude that is independent of the amount of tissue factor as trigger. ${ }^{23}$ The thrombin amplitude in this stationary phase $\left(A_{\mathrm{st}}\right)$ and the longitudinal rate of thrombin propagation $\left(V_{\mathrm{t}}\right)$ were calculated.

Calibrated automated thrombography

CAT was performed with adult and cord blood samples as reported previously. ${ }^{24}$ Thrombin generation was triggered with $5 \mathrm{pM}$ tissue factor in the presence of $4 \mu \mathrm{M}$ phospholipids using the Thrombinoscope PPP reagent (Diagnostica Stago, Asnières sur 
Seine Cedex, France). Thrombin traces were recorded and thrombin generation parameters were calculated with the Thrombinoscope software (Diagnostica Stago, Asnières sur Seine Cedex, France).

\section{Routine parameters}

PT, aPTT, and FIl were determined with one-stage clotting assays using an ACL Top 350 coagulation analyzer with Hemosil reagents (Instrumentation Laboratory, Bedford, MA). Antithrombin was measured with a chromogenic method (Instrumentation Laboratory, Bedford, MA), and TFPI was measured with ELISA (Sekisui Diagnostics, Lexington, MA).

\section{Statistics}

All calculations were done using GraphPad Prism 6.0 (GraphPad software, San Diego, CA). Values are given as mean \pm standard deviation or median (range) if distribution was not normal. Differences between groups were analyzed using analysis of variance and post hoc tests. Corrections for multiple comparisons were made via the Holm-Šídák method $(a=0.05)$, and multiplicity-adjusted $P$ values were calculated for each comparison. Differences between demographic data of neonatal cohorts were tested with Mann-Whitney U-test. Correlations between CAT and thrombodynamics parameters were calculated with Pearson's coefficients.

\section{RESULTS}

Thrombodynamics

Dynamics of clot growth and thrombin generation in a representative adult and neonatal sample are depicted in Fig. 1. Lag time until clot formation was comparable in adult, venous neonatal, and cord blood samples (adult: $0.83 \pm 0.07 \mathrm{~min}$; neonatal: $0.81 \pm 0.07 \mathrm{~min}$; cord: $0.80 \pm 0.08 \mathrm{~min} ; P=0.843)$. Interestingly, the initial (tissue factor-dependent) rate of clot formation $\left(V_{i}\right)$ was higher in venous neonatal and cord blood samples compared to adult samples (adult: $60.7 \pm 3.9 \mu \mathrm{m} / \mathrm{min}$; neonatal: $66.8 \pm 3.9 \mu \mathrm{m} / \mathrm{min}$; cord: $68.1 \pm 3.3 \mu \mathrm{m} / \mathrm{min} ; P<0.001$; Fig. 2a). The stationary (tissue factor-independent) rate of clot formation $\left(V_{\mathrm{s}}\right)$ was comparable in all study groups (adult: $35.8 \pm 8.5 \mu \mathrm{m} / \mathrm{min}$; neonatal: $37.0 \pm 4.6 \mu \mathrm{m} / \mathrm{min}$; cord: $36.0 \pm 5.2 \mu \mathrm{m} / \mathrm{min} ; P=0.834$ Fig. $2 \mathrm{~b}$ ). The clot size after $30 \mathrm{~min}$ was comparable in all groups (adult: $1203 \pm 135 \mu \mathrm{m}$; neonatal: 1281 $\pm 91 \mu \mathrm{m}$; cord: $1311 \pm 148 ; P=0.224)$. The optical clot density was comparable in all study groups (adult: $22074 \pm 2143 \mathrm{AU}$; neonatal: $22264 \pm 2863 \mathrm{AU}$; cord: $21934 \pm 2625 \mathrm{AU} ; P=0.435$ ). The amount of thrombin generated within the clot during the measurement was substantially lower in venous neonatal and cord blood samples. Accordingly, the stationary thrombin amplitude $\left(A_{s t}\right)$ was significantly lower in neonates and cord blood compared to adults (adult: $41.9 \pm 11.2 \mathrm{AU} / \mathrm{l}$; neonatal: $22.6 \pm 10.2 \mathrm{AU} / \mathrm{l}$; cord: $23.6 \pm 9.7 \mathrm{AU} / \mathrm{l} ; P<$ 0.001 ; Fig. 2c). Interestingly, the rate of thrombin propagation $\left(V_{\mathrm{t}}\right)$ was comparable in all study groups (adult: $27.2 \pm 4.2 \mu \mathrm{m} / \mathrm{min}$; neonatal: $27.9 \pm 2.9 \mu \mathrm{m} / \mathrm{min}$; cord: $27.6 \pm 3.4 \mu \mathrm{m} / \mathrm{min} ; \quad P=0.862$; Fig. 2d). Thrombodynamics results of each neonate according to postnatal age are depicted in Table S2.

\section{Calibrated automated thrombography}

Thrombin generation traces of cord blood samples exhibited a shorter lag time and time to peak than those of adult samples $(P<$ 0.001 ; Table 2). Peak height, ETP, and velocity index were significantly lower in cord blood than in adult samples (peak height: $P<0.001$; ETP: $P<0.001$; velocity index: $P<0.01$; Table 2 ).

Routine coagulation parameters

Venous neonatal and cord blood samples exhibited a longer PT than adult samples, but remained within the normal range of adults (Table 3). APTT values in venous neonatal and cord blood samples were higher than the normal range of adults, but within a
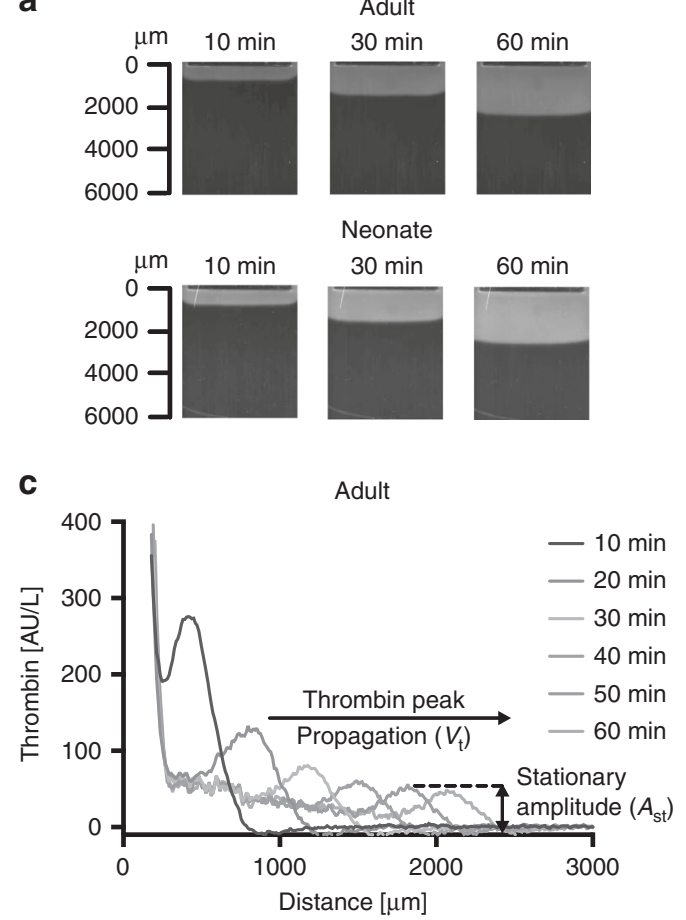

b
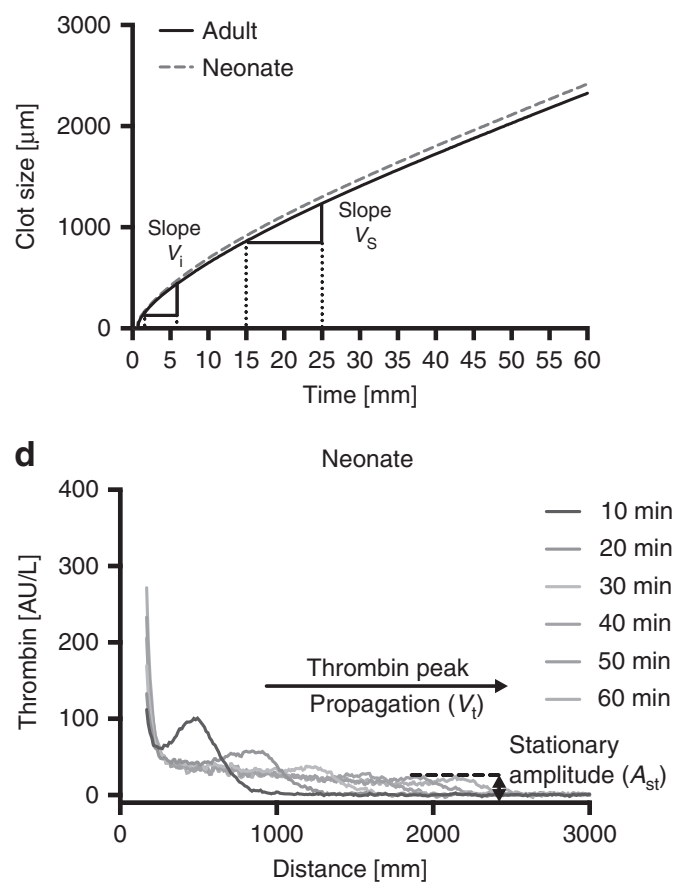

Fig. 1 Thrombodynamics data from a representative adult and neonatal sample. Subfigures show optical presentation of the fibrin clot (light) in plasma (dark) after 10,30, and $60 \mathrm{~min}(\mathbf{a})$, resulting clot growth traces indicating initial rate of clot formation $\left(V_{\mathrm{i}}\right)$ and stationary rate of clot formation $\left(V_{s}\right)(\mathbf{b})$, and thrombin distribution within the clot in 10 min-intervals in the adult sample (c) and the neonatal sample (d). Thrombin distribution is plotted in amount of thrombin over distance from the tissue factor bearing insert 

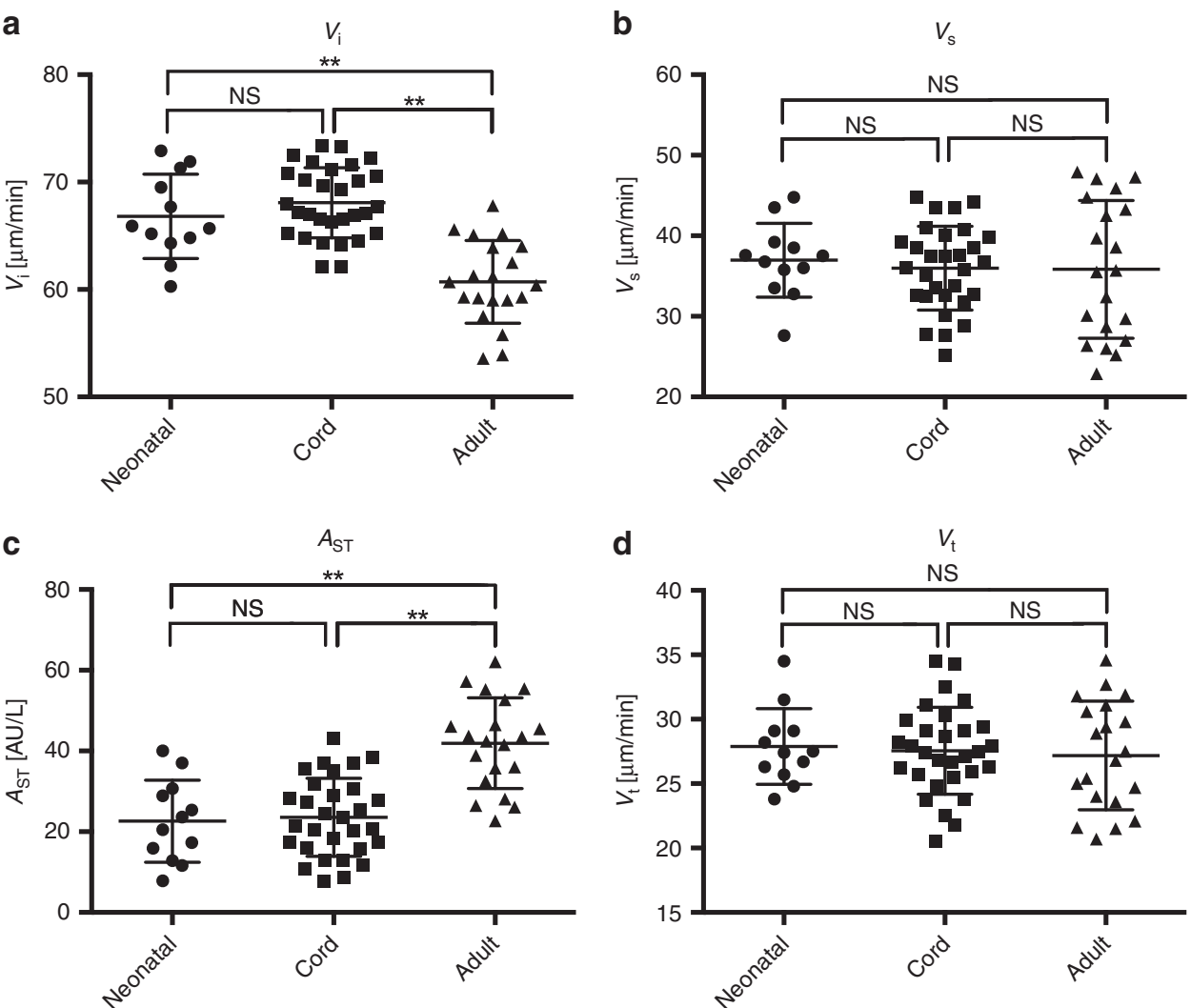

Fig. 2 Distribution of thrombodynamics parameters in adult samples $(N=20)$, venous neonatal samples $(N=12)$, and samples from cord blood $(N=30)$. Fibrin clot propagation is characterized by initial rate of clot formation $\left(V_{i}\right)(\mathbf{a})$ and stationary rate of clot formation $\left(V_{s}\right)(\mathbf{b})$. Thrombin generation is characterized by the stationary thrombin amplitude $\left(A_{\mathrm{st}}\right)(\mathbf{c})$ and thrombin peak propagation $\left(V_{\mathrm{t}}\right)(\mathbf{d}) .{ }^{* *} P<0.001 ; \mathrm{NS}=$ no significance

Table 2. Thrombin generation (CAT) parameters of adult $(N=20)$ and cord blood samples $(N=30)$

\begin{tabular}{lcc}
\hline & \multicolumn{1}{l}{ Adult } & \multicolumn{1}{c}{ Cord } \\
\hline Lag time [min] & $2.63 \pm 0.43$ & $1.71 \pm 0.58^{* *}$ \\
Time to peak [min] & $6.12 \pm 1.19$ & $3.94 \pm 0.74^{* *}$ \\
Peak height [nM] & $272.4 \pm 77.7$ & $191.5 \pm 29.4^{* *}$ \\
ETP [nM*min] & $1709 \pm 315$ & $934 \pm 121^{* *}$ \\
Velocity index [nM/min] & $78.2 \pm 40.6$ & $83.7 \pm 35.2$ \\
\hline $\begin{array}{l}\text { Data are depicted in mean } \pm \text { standard deviation. Asterisks indicate } \\
\left.\text { significant differences between cord and adult samples }{ }^{* *} P<0.001\right) \\
\text { CAT calibrated automated thrombography, ETP endogenous thrombin } \\
\text { potential }\end{array}$ \\
\hline
\end{tabular}

the normal range of healthy neonates (Table 3). Plasma levels of FII, antithrombin, and TFPI were substantially lower in venous neonatal and cord blood samples than in adult samples (Table 3).

\section{Correlations}

Correlations analyses were performed with cord blood and adult samples. The stationary rate of clot formation $\left(V_{s}\right)$ correlated with the longitudinal rate of thrombin propagation $\left(V_{t}\right)(r=0.842 ; P<$ 0.001 ) (Figure $\mathrm{S} 1 \mathrm{a})$, but only weakly with intra-clot thrombin levels $\left(A_{\mathrm{st}}\right)(r=0.355 ; P<0.05)$ (Figure S1b). The ETP from CAT correlated with intra-clot thrombin levels $\left(A_{\mathrm{st}}\right)(r=0.800 ; P<0.001)$ (Figure S1c), but not with longitudinal rate of thrombin propagation $\left(V_{\mathrm{t}}\right)(r=0.194 ; P=0.177)$ (Figure $\left.\mathrm{S} 1 \mathrm{~d}\right)$.

\section{DISCUSSION}

Investigations on peculiarities of neonatal coagulation and the development of age-appropriate assays depicting the clinical picture of healthy infants are crucial, because such studies provide the basis for distinguishing and treating infants with thrombotic or hemorrhagic tendencies. Several assays have been employed to evaluate the balance between clotting factors and inhibitors in neonates. ${ }^{12,15,25}$ However, all of them suffered from inadequacies regarding the amount and distribution of tissue factor, which hampered conclusions on in vivo conditions. In this study, neonatal clot propagation has been observed in a setting that overcomes these inadequacies for the first time.

Research on neonatal coagulation is always hampered by low numbers of recruitable participants and small amounts of sample material. Hence, we used thrombodynamics to investigate spatial fibrin clot formation and intra-clot thrombin levels of venous blood from a small cohort of neonates and substantiated our results with a larger number of cord blood samples from uncomplicated deliveries. Despite known limitations of cord blood as a surrogate for neonatal blood, both cohorts were comparable in PT, APTT, FII, AT, and TFPI levels (Table 3). Additionally, cord blood samples could be analyzed with CAT due to sufficient amounts of sample material. Differences in thrombin generation parameters between cord blood and adult cohorts were expected and comparable to previous studies. ${ }^{15,25}$ We found shorter lag time and time to peak, but lower peak height and ETP in cord blood. Shorter lag time and time to peak are potentially caused by lower amounts of TFPI and antithrombin, while peak height and ETP are limited by lower FIl levels in neonates (Table 3). However, representativeness of thrombin generation traces for the clinical phenotype of neonates is ambiguous, as the respective contribution of tissue 
Table 3. Routine coagulation parameters of adult $(N=20)$, neonatal samples $(N=12)$, and cord blood samples $(N=30)$

\begin{tabular}{lccc}
\hline & Adult & Neonatal & Cord \\
\hline PT [s] & $12.0 \pm 0.7(9.4-12.5)$ & $13.1 \pm 0.7^{* *}(11.6-14.4)$ & $12.8 \pm 1.1^{*}(11.6-14.4)$ \\
APTT [s] & $33.0 \pm 3.8(27.5-36.3)$ & $43.8 \pm 6.0^{* *}(27.6-45.6)$ & $42.6 \pm 7.8^{* *}(27.6-45.6)$ \\
FII [\%] & $94.1 \pm 14.8(75.2-132)$ & $48.6 \pm 6.7^{* *}(44,8-74,3)$ & $52.1 \pm 8.4^{* *}(44,8-74,3)$ \\
Antithrombin [\%] & $106.1 \pm 12.6(83.2-125.7)$ & $51.1 \pm 7.2^{* *}(32.8-62.8)$ & $49.8 \pm 12.2^{* *}(32.8-62.8)$ \\
TFPI [ng/ml] & $54.4 \pm 17.2(50-100)$ & $19.1 \pm 5.6^{* *}$ & $17 . \pm 10.9^{* *}$ \\
\hline
\end{tabular}

Data are depicted in mean \pm standard deviation. Asterisks indicate significant differences between neonatal/cord and adult samples $\left({ }^{*} P<0.05 ;{ }^{*} P<0.001\right)$

Adult and neonatal reference ranges are depicted in brackets

PT prothrombin time, APTT activated partial thromboplastin time, Fll factor II, TFPI tissue factor pathway inhibitor

factor-dependent and -independent processes to overall thrombin generation is varying with the employed tissue factor concentration and cannot be evaluated in spatial separation. Furthermore, we do not know whether the observed differences in thrombin generation between adults and cord blood samples translate into differences in bleeding cessation.

We used the thrombodynamics assay featuring immobilized tissue factor and detection of spatial clot formation in combination with intra-clot thrombin levels to gain higher representativeness for in vivo conditions. We employed this assay in all three sample types (venous neonatal blood, cord blood, adult blood). While no differences were found between samples from neonatal blood and from cord blood, both study cohorts were significantly different to samples from adult blood. Thrombodynamics data in the two cohorts representing neonates fit well with the postulated concept of balanced hemostasis wherein lower inhibitor levels even out lower levels of clotting factors. Based on the neonatal clinical picture and the sum of laboratory parameters, we hypothesized that spatial clot propagation was comparable in neonatal and adult samples. Interestingly, tissue factor-dependent processes corresponding to the initiation phase of hemostasis (lag time, $V_{i}$ ) occur at even faster rates in neonates than in adult samples. This can be explained by normal FVII levels in neonates ${ }^{5}$ in combination with lower TFPI levels (Table 3) facilitating tissue factor-dependent onset of thrombin generation. The stationary rate of clot formation $\left(V_{s}\right)$ was comparable in neonates and adults, which confirmed our hypothesis. At this stage the clot front is not in proximity of immobilized tissue factor. Hence, the clot propagation is largely dominated by tissue factor-independent processes of the propagation phase. The comparable stationary rate shows that in this phase the balance between coagulationdriving and -inhibiting factors is comparable in neonates and adults despite differing absolute levels.

Interestingly, our results differ somewhat from the data shown by Koltsova et al. ${ }^{20}$ While data for $V_{\mathrm{i}}$ were similar in both studies, $V_{\mathrm{s}}$ was lower in adults compared to neonates in the Koltsova study, but comparable in our study. We hypothesize that this discrepancy occurred due to methodological differences. We added phospholipids to our plasma samples in order to measure intraclot thrombin levels, while Koltsova et al. ${ }^{20}$ only measured fibrin clot formation without phospholipids. As we have recently shown, neonatal coagulation is less dependent on phospholipids than adult coagulation. ${ }^{26}$ Hence, omittance thereof in the Koltsova study potentially affected the adult cohort more than the neonatal cohort, which may explain aforementioned discrepancy.

Surprisingly, the amount of thrombin generated within the clot was significantly lower in neonatal samples than in adult samples despite comparable rates of clot propagation. This is reflected in a significantly lower amplitude of stationary thrombin formation $\left(A_{\text {st }}\right)$ and could be a result of lower Fll levels. The longitudinal rate of thrombin propagation $\left(V_{t}\right)$, on the other hand, was comparable in neonatal and adult samples. This parameter shows how fast the thrombin peak at the clot front propagates.
Correlation analyses were performed with data from cord blood and adult blood. $V_{\mathrm{t}}$ but not $A_{\mathrm{st}}$ correlated highly with the stationary rate of fibrin clot propagation $\left(V_{s}\right)$. This finding indicates that the rate of longitudinal thrombin propagation $\left(V_{t}\right)$ and not the overall amount of thrombin $\left(A_{\mathrm{st}}\right)$ determines the rate of fibrin clot formation and explains why neonatal samples can have comparable clot propagation at lower thrombin levels. We postulate that the rate at which very small amounts of thrombin can be formed at the edge of the clot front is the actual decisive factor for the rate of clot propagation. This notion is supported by a previous study showing close to normal clot growth velocity despite significantly decreased thrombin generation with low Fll levels. ${ }^{27}$ This crucial relation between thrombin and fibrin clot propagation cannot be characterized with CAT because it depends on immobilized tissue factor to manifest. Therefore, ETP correlated only with $A_{\text {st }}$ but not with $V_{\text {t }}$.

We did not add thrombomodulin to our thrombodynamics experiments, because this activator of the protein C/S system is not a plasma component, but localized on the surface of intact endothelial cells surrounding the damaged area. Hence, it is not part of the tissue factor exposed microenvironment modeled by the thrombodynamics assay.

Balanced hemostasis despite lower clotting factor levels is also observed in patients with cirrhosis. ${ }^{28}$ Interestingly, these patients exhibit normal spatial fibrin clot formation similar to neonates. ${ }^{29}$ Cirrhosis is accompanied with a higher incidence rate of bleeding as well as thrombosis, which is attributable to the increased fragility of a hemostatic balance at lower levels. This increased fragility is potentially also present in neonates and should be taken into account.

A limitation of this study is the recruitment during follow-up of prolonged neonatal jaundice. We want to point out that these infants exhibited clinically inconspicuous coagulation and routine coagulation parameters within neonatal normal range. Lower antithrombin levels have been reported in neonates with severe jaundice, but our cohort exhibited antithrombin levels of healthy neonates. ${ }^{15,30}$ To substantiate our findings, we collected a larger number of cord blood samples from uncomplicated deliveries. Cord blood provides only limited representativeness as a surrogate for neonatal blood, but both study cohorts were comparable in all employed coagulation assays. Hence, our neonatal cohort can be considered healthy from a hemostaseological perspective. A neonatal blood draw without any other diagnostic incentive is hardly ethically justifiable. Limited amounts of sample material prohibited a full panel of all clotting factors and inhibitors. However, normal ranges in newborns have been well studied in aforementioned literature.

\section{CONCLUSION}

Taken together, we have shown that neonates and adults exhibit comparable clot growth when immobilized tissue factor is used to trigger coagulation, albeit intra-clot thrombin levels are 
significantly lower in neonates. Our data show that the hemostatic balance in neonates and adults is similar despite lower clotting factor levels in healthy neonates, which explains the wellfunctioning hemostasis in this patient group. However, due to lower absolute levels of respective factors and inhibitors, neonatal hemostasis may be easily dysbalanced by infection and inflammation. This fragility and the underlying hemostatic peculiarities should be kept in mind when treating neonatal hemorrhage or thrombosis.

\section{ACKNOWLEDGEMENTS}

We thank Y. Gallistl for secretarial support. This work was supported financially by CSL Behring and Shire. Reagents and rental devices were provided by Hemacore and Coachrom.

\section{AUTHOR CONTRIBUTIONS}

H.H. contributed to conception and design of the study, data interpretation, and manuscript drafting. E.Z., S.P. and B.L. performed data acquisition and analyses. E.-C.W, S.G. and W.M. contributed to conception and design, and revised the article critically for intellectual content. A.S. contributed to conception and design, data interpretation, manuscript drafting, and gave final approval of the version to be published.

\section{ADDITIONAL INFORMATION}

The online version of this article (https://doi.org/10.1038/s41390-019-0531-4) contains supplementary material, which is available to authorized users.

Competing interests: The authors declare no competing interests.

Publisher's note: Springer Nature remains neutral with regard to jurisdictional claims in published maps and institutional affiliations.

\section{REFERENCES}

1. Manco-Johnson, M. J. Hemostasis in the neonate. NeoReviews 9, e119-e123 (2008).

2. Monagle, P. \& Massicotte, P. Developmental haemostasis: secondary haemostasis. Semin. Fetal Neonatal Med. 16, 294-300 (2011).

3. Revel-Vilk, S. The conundrum of neonatal coagulopathy. Hematol. Am. Soc. Hematol. Educ. Program 2012, 450-454 (2012).

4. DelVecchio, A., Latini, G., Henry, E. \& Christensen, R. D. Template bleeding times of 240 neonates born at 24 to 41 weeks gestation. J. Perinatol. 28, 427-431 (2008).

5. Andrew, M. et al. Development of the human coagulation system in the full-term infant. Blood 70, 165-172 (1987).

6. Cvirn, G. et al. Alpha 2-macroglobulin enhances prothrombin activation and thrombin potential by inhibiting the anticoagulant protein $\mathrm{C} /$ protein $\mathrm{S}$ system in cord and adult plasma. Thromb. Res. 105, 433-439 (2002).

7. Cvirn, G., Gallistl, S. \& Muntean, W. Effects of alpha(2)-macroglobulin and antithrombin on thrombin generation and inhibition in cord and adult plasma. Thromb. Res. 101, 183-191 (2001).

8. Fritsch, P. et al. Thrombin generation in factor VIII-depleted neonatal plasma: nearly normal because of physiologically low antithrombin and tissue factor pathway inhibitor. J. Thromb. Haemost. 4, 1071-1077 (2006).
9. Cvirn, G., Gallistl, S., Rehak, T., Jurgens, G. \& Muntean, W. Elevated thrombinforming capacity of tissue factor-activated cord compared with adult plasma. J. Thromb. Haemost. 1, 1785-1790 (2003).

10. Tripodi, A. et al. Normal thrombin generation in neonates in spite of prolonged conventional coagulation tests. Haematologica 93, 1256-1259 (2008).

11. Roschitz, B., Sudi, K., Kostenberger, M. \& Muntean, W. Shorter PFA-100 closure times in neonates than in adults: role of red cells, white cells, platelets and von Willebrand factor. Acta Paediatr. 90, 664-670 (2001).

12. Edwards, R. M., Naik-Mathuria, B. J., Gay, A. N., Olutoye, O. O. \& Teruya, J. Parameters of thromboelastography in healthy newborns. Am. J. Clin. Pathol. 130, 99-102 (2008).

13. Hemker, H. C. et al. Calibrated automated thrombin generation measurement in clotting plasma. Pathophysiol. Haemost. Thromb. 33, 4-15 (2003).

14. Kremers, R. M. W., Peters, T. C., Wagenvoord, R. J. \& Hemker, H. C. The balance of pro- and anticoagulant processes underlying thrombin generation. J. Thromb. Haemost. 13, 437-447 (2015).

15. Cvirn, G., Gallistl, S., Leschnik, B. \& Muntean, W. Low tissue factor pathway inhibitor (TFPI) together with low antithrombin allows sufficient thrombin generation in neonates. J. Thromb. Haemost. 1, 263-268 (2003).

16. Duchemin, J., Pan-Petesch, B., Arnaud, B., Blouch, M.-T. \& Abgrall, J.-F. Influence of coagulation factors and tissue factor concentration on the thrombin generation test in plasma. Thromb. Haemost. 99, 767-773 (2008).

17. Hoffman, M. 3rd DMM. A cell-based model of hemostasis. Thromb. Haemost. 85, 958-965 (2001).

18. Panteleev, M. A. et al. Spatial propagation and localization of blood coagulation are regulated by intrinsic and protein $C$ pathways, respectively. Biophys. J. 90, 1489-1500 (2006).

19. Tuktamyshov, R. \& Zhdanov, R. The method of in vivo evaluation of hemostasis: spatial thrombodynamics. Hematology 20, 584-586 (2015).

20. Koltsova, E. M. et al. Impaired platelet activity and hypercoagulation in healthy term and moderately preterm newborns during the early neonatal period. Pediatr. Res. 85, 63-71 (2019).

21. Schlagenhauf, A., Schweintzger, S., Birner-Gruenberger, R., Leschnik, B. \& Muntean, W. Newborn platelets: lower levels of protease-activated receptors cause hypoaggregability to thrombin. Platelets 21, 641-647 (2010).

22. Schlagenhauf, A. et al. Polyphosphate in neonates: less shedding from platelets and divergent prothrombotic capacity due to lower TFPI levels. Front. Physiol. 8, 586 (2017).

23. Dashkevich, N. M. et al. Thrombin activity propagates in space during blood coagulation as an excitation wave. Biophys. J. 103, 2233-2240 (2012).

24. Cimenti, C. et al. Only minor changes in thrombin generation of children and adolescents with type 1 diabetes mellitus-a case-control study. Thromb. Res. 148, 45-49 (2016)

25. Schweintzger, S. et al. Microparticles in newborn cord blood: slight elevation after normal delivery. Thromb. Res. 128, 62-67 (2011).

26. Haidl, H. et al. Neonatal thrombocytopenia: thrombin generation in presence of reduced platelet counts and effects of rFVIla in cord blood. Sci. Rep. 9, 8014 (2019).

27. Kuprash, A. D. et al. Sensitivity and robustness of spatially dependent thrombin generation and fibrin clot propagation. Biophys. J. 115, 2461-2473 (2018).

28. Kremers, R. M. W. et al. Decreased prothrombin conversion and reduced thrombin inactivation explain rebalanced thrombin generation in liver cirrhosis. PLOS ONE 12, e0177020 (2017).

29. Potze, W., Adelmeijer, J., Porte, R. J. \& Lisman, T. Preserved clot formation detected by the thrombodynamics analyzer in patients with cirrhosis. Thromb. Res. 135, 1012-1016 (2015).

30. Baptista-González, H., Gutiérrez-Landeros, F., Rosenfeld-Mann, F. \& TruebaGómez, R. Changes of coagulation inhibitors and fibrinolysis system in newborn infants with transitory neonatal cholestasis. Ann. Hepatol. 3, 26-29 (2004). 